\title{
Comunidade de insetos visitantes florais de Ocimum selloi Benth (Lamiaceae) em Santa Cruz do Sul, RS, Brasil
}

\author{
Karine Schoeninger $^{1 *}$ \\ Alexandre Somavilla ${ }^{2}$ \\ Andreas Köhler ${ }^{1}$ \\ ${ }^{1}$ Laboratório de Entomologia, Universidade de Santa Cruz do Sul \\ Avenida Independência, 2293, CEP 96815-900, Santa Cruz do Sul - RS, Brasil \\ 2Instituto Nacional de Pesquisas da Amazônia (INPA) \\ Coordenação de Pesquisas em Entomologia \\ Caixa Postal 478, CEP 69011-970, Manaus - Brasil \\ *Autor para correspondência \\ kackes.s@gmail.com
}

Submetido em 31/05/2011

Aceito para publicação em 14/10/2011

\section{Resumo}

Este trabalho objetivou listar a comunidade de visitantes florais de Ocimum selloi, indicar os potenciais polinizadores e seus padrões de visitação. A coleta dos visitantes florais ocorreu no ano de 2007, em área antropofizada, sendo analisada a constância e a dominância dos táxons. Coletou-se um total de 653 insetos, pertencentes a sete ordens; Hymenoptera, com 370 indivíduos, foi o grupo mais representativo. Foram identificadas 36 famílias, sendo 12 de Hymenoptera e oito de Diptera, as mais diversas. Apidae foi a família com maior constância e dominância compreendida, principalmente, pela abelha Apis mellifera L., 1758 ( $\mathrm{n}=$ 223), seguida de Halictidae $(\mathrm{n}=107)$. A maior atividade forrageira foi observada no horário entre as $9 \mathrm{~h}$ e $11 \mathrm{~h}$ da manhã. Constatou-se que $A$. mellifera foi o principal visitante floral e potencial polinizador de $O$. selloi.

Palavras-chave: Apis mellifera, Entomofauna, Hymenoptera, Polinização

\section{Abstract}

Community of floral visiting insects of Ocimum selloi Benth (Lamiaceae) in Santa Cruz do Sul, Rio Grande do Sul, Brazil. This paper aimed to list the community of floral visitors of Ocimum selloi, indicate the potential pollinators, and their patterns of visitation. The collection of flower visitors was carried out in 2007, in an anthroposophical area, with analysis of constancy and dominance of taxa. A total of 653 insects was collected, belonging to seven orders; Hymenoptera, with 370 individuals, was the most representative group. Thirty six families were identified, being 12 of Hymenoptera and eight of Diptera the most diverse ones. Apidae was the family which showed the greatest constancy and dominance, especially through the bee Apis mellifera L., 1758 $(\mathrm{n}=223)$, followed by Halictidae $(\mathrm{n}=107)$. Most of the foraging activity was observed between 9a.m. and 11a.m. It was found that $A$. mellifera was the main floral visitor and potential pollinator of $O$. selloi.

Key words: Apis mellifera, Entomofauna, Hymenoptera, Pollination 


\section{Introdução}

O gênero Ocimum L., pertencente à família Lamiaceae e compreende aproximadamente 30 espécies de ervas e subarbustos que estão dispersos pelas regiões tropicais e subtropicais da Ásia, África, Américas Central e do Sul (PATON, 1992). O gênero apresenta grande diversidade de espécies, sendo que no Brasil são encontradas doze que ocorrem principalmente na região Sul e Sudeste (MATOS, 1998). São plantas com ciclos anuais ou perenes, dependendo do local em que são cultivadas e da aplicação comercial de seus subprodutos (BLANK et al., 2004). Na culinária, utilizam-se as folhas frescas ou desidratadas como condimento, já na produção de fitoterápicos são obtidos terpenóides e compostos fenólicos das folhas, inflorescências e sementes (SIMON et al., 1990; JAVANMARDI et al., 2002).

Ocimum também tem despertado a atenção de pesquisadores por apresentar espécies que possuem óleos essenciais, além de apresentar atividades aromáticas, medicinais e condimentares (SIMON et al., 1990; MORALES; SIMON, 1996).

Espécies de Ocimum ocorrem em diversos ambientes naturais e antropizados e foram apontadas como fonte de recursos para várias guildas de insetos, dentre eles nectarívoros e herbívoros (VINCENT et al., 2002).

No Brasil, em estudos anteriores referentes a abelhas encontraram uma grande variação na sensibilidade das espécies à urbanização e uma considerável redução da diversidade em ambientes altamente urbanizados (LAROCA et al., 1982; BORTOLI; LAROCA, 1997; KNOLL et al., 1993). Supõe-se, geralmente, que a urbanização (não considerando desertos), reduz a complexidade de habitat, principalmente através da redução da cobertura vegetal natural. Consequentemente, pode-se prever que áreas menos urbanizadas são estruturalmente mais complexas. Por estas razões, também pode ser previsto que áreas menos urbanizadas apóiam uma maior abundância e riqueza de espécies de vespas e abelhas (ZANETTE et al., 2005).

Ocimum selloi Benth conhecido popularmente como manjericão ou alfavaca é um subarbusto ereto com caule quadrangular característico, podendo atingir até $1,20 \mathrm{~cm}$ de altura; suas folhas são simples e opostas, com margem serrilhada, ápice acuminado e base atenuada, medindo até $5 \mathrm{~cm}$ de comprimento por até $2,5 \mathrm{~cm}$ de largura; as flores são pequenas, de cor branca, dispostas em racemos terminais curtos; os frutos são aquênios de cor escura que não se separam facilmente da semente. (MORHY, 1973; LORENZI; MATOS, 2002).

As flores apresentam atributos florais relacionados à síndrome floral de melitofilia (FAEGRI; VAN DER PIJL, 1980), tais como corola pouco tubulosa, odor doce, pequena distância entre a câmara nectarífera e os verticilos férteis e antese diurna. Estudos preliminares sobre a biologia floral e sistema reprodutivo de espécies do gênero Ocimum estão representados pelos trabalhos pioneiros de Raju (1989), das espécies $O$. americanum L. e $O$. basilicum L. e em estudos realizados com $O$. officinalis L. por Almeida et al. (2004), os quais afirmam que estas espécies são consideradas predominantemente autógamas, mas que também podem reproduzir-se por fecundação cruzada. De acordo com estudos realizados por Khosla (1986), as espécies da família Lamiaceae têm uma estrutura floral favorável à polinização por abelhas.

Os insetos possuem destacada importância nos diversos processos de interações entre plantas e animais, principalmente nos de polinização. Os principais grupos de insetos visitantes florais são abelhas, vespas, besouros, moscas, formigas e borboletas. Estes grupos de insetos geralmente visam à obtenção de pólen, néctar ou óleos (CONSTANTINO et al., 2002). Além disso, grande parte dos trabalhos sobre visitantes florais aponta as abelhas como principais membros nas comunidades de polinizadores mais eficazes (GRISWOLD et al., 1995; WILMS et al., 1997).

Existem pelo menos duas estratégias que garantiriam o acesso dos polinizadores aos recursos florais disponíveis. Na primeira, os polinizadores exploram os recursos florais de diferentes espécies de plantas (PLEASANTS, 1980). Já na segunda, os polinizadores buscam recursos em diferentes horas do dia ou diferentes períodos de uma estação (GINSBERG, 1983).

Sendo assim, na ausência de estudos sobre comunidades de insetos visitantes florais de $O$. sello $i$ 
no estado do Rio Grande do Sul, este trabalho tem por objetivo listar as espécies de insetos visitantes florais, indicar seus potenciais polinizadores, além de apontar a preferência dos horários de forrageio nas flores.

\section{Material e Métodos}

O estudo foi realizado em uma área cultivada de O. selloi, no campus da Universidade de Santa Cruz do Sul - UNISC, situada nas coordenadas geográficas $29^{\circ} 41^{\prime} 52,25^{\prime}$ 'S e $52^{\circ} 26^{\prime} 10,31^{\prime \prime} \mathrm{W}$, cujo local compreende um ambiente antropizado, cercado de prédios e com ausência de vegetação nativa. A cidade de Santa Cruz do Sul pertence à região da Encosta Inferior do Nordeste do estado do Rio Grande do Sul (FORTES, 1979), na unidade geomorfológica correspondente aos patamares da Serra Geral (COLLISCHONN, 2001). A vegetação predominante é a Floresta Estacional Decidual (IBGE, 1986) e o clima, segundo a classificação de Köppen (1948), é do tipo Cfa (Subtropical úmido).

A coleta dos visitantes florais foi realizada em quatro dias, entre $7 \mathrm{~h}$ e $17 \mathrm{~h}$, no mês de abril de 2007 . Foram coletados apenas os insetos pousados nas flores, separadamente a cada período de $1 \mathrm{~h}$, totalizando $40 \mathrm{~h}$ amostradas, através do uso de redes entomológicas pequenas $(50 \mathrm{~cm}$ de diâmetro) confeccionadas com tecido organza cristal branco para não danificar as flores. Imediatamente após a coleta, os espécimes foram depositados em câmeras mortíferas contendo acetato de etila e posteriormente alfinetados, identificados até nível de ordem através de chave dicotômica segundo Azevedo Filho e Prates Jr. (2005) e depositados na Coleção Entomológica de Santa Cruz do Sul (CESC). A identificação dos indivíduos pertencentes à ordem Hymenoptera seguiu a referência de Silveira et al. (2002) e para as demais ordens seguiu-se a de Triplehorn e Johnson (2011). Durante todo o período de coleta, foi acompanhado o horário, bem como a temperatura, com o auxílio de um termômetro, sendo esta registrada a cada hora.

A dominância dos táxons coletados foi calculada através da fórmula $\mathrm{D}=(\mathrm{i} / \mathrm{t}) \times 100$, onde $\mathrm{D}=$ dominância $(\%), i=$ total de indivíduos de uma determinada espécie e $t=$ total de indivíduos coletados, sendo que $\mathrm{D}=>10 \%$
Eudominante, $\mathrm{D}=5-10 \%$ Dominante, $\mathrm{D}=2-5 \%$ Subdominante, $\mathrm{D}=1-2 \%$ Recessiva e $\mathrm{D}=<1 \%$ Rara (PALISSA et al., 1979).

A constância dos táxons coletados foi calculada através da fórmula $\mathrm{C}=\mathrm{p} \times 100 / \mathrm{N}$, onde $\mathrm{C}=$ constância $(\%), p=$ número de coletas, que no presente estudo, ocorreu o táxon em questão, considerou-se os números de horas que a espécie foi coletada, e $N=$ número total de coletas efetuadas; sendo agrupadas como $\mathrm{C}=>50 \%$ Constante, $\mathrm{C}=25-50 \%$ Acessória e $\mathrm{C}=<25$ Acidental (BODENHEIMER, 1955).

\section{Resultados}

Foi coletado um total de 653 insetos, pertencentes a sete ordens, sendo Hymenoptera com 370 indivíduos o grupo com maior número de espécimes, seguida por Hemiptera $(n=163)$, Diptera $(n=56)$, Coleoptera $(n=31)$, Lepidoptera (24), Orthoptera $(n=5)$ e Odonata $(n=4)$.

Foram identificadas 36 famílias, sendo 12 de Hymenoptera, oito de Diptera e sete de Hemiptera, as mais diversas; além de três famílias de Coleoptera, Odonata e Orthoptera. Os espécimes de Lepidoptera coletados não puderam ser identificados devido às dificuldades na identificação. Em Hymenoptera, identificaram-se 38 táxons visitantes florais, sendo que Halictidae apresentou a maior diversidade de espécies (13), seguida de Megachilidae (6) e Apidae e Vespidae (5) (Tabela 1).

As famílias de Hymenoptera que se destacaram pelo maior número de espécimes coletados foram Apidae ( $\mathrm{n}=$ $228)$, seguida por Halictidae $(n=107)$; estes dois táxons foram considerados Eudominantes, por apresentarem mais de $10 \%$ do total de indivíduos coletados.

Miridae com 87 e Cicadelidae com 65, ambos pertencentes à ordem Hemiptera, também foram coletadas em grande quantidade e classificadas como Eudominantes. Sendo assim, as famílias Apidae, Halictidae, Miridae e Cicadelidae representaram 77\% do total de insetos determinados.

Apis mellifera L. (Apidae) juntamente com Miridae sp. 1 (Hemiptera) foram as únicas espécies eudominantes e constantes no presente estudo (Tabela 1). Observa- 
se na Tabela 1 que outros himenópteros foram pouco freqüentes e abundantes, isso, possivelmente, se deve à competitividade de $A$. mellifera, visto que no período de visitação desta abelha a presença de outros insetos nas flores foi baixa.

A análise dos táxons por horário (Figura 1) mostrou maior frequência de visitação às $10 \mathrm{~h}(\mathrm{n}=$ $103)$, seguida por $9 h(n=84)$ e $8 h(n=82)$. Verificou- se uma maior presença dos insetos visitantes florais pela parte da manhã, com temperaturas entre 24 e $26^{\circ}$. Em Hymenoptera, os horários de maior atividade nas flores foram entre $10 \mathrm{~h}$ e $13 \mathrm{~h}$. Em especial dentro de Halictidae, na qual observa-se em Dialictus sp. 1 e Augochloropsis sp. 1 a preferência pelo horário das $10 \mathrm{~h}$ tendo sido coletados 10 e seis indivíduos neste período, respectivamente.

TABELA 1: Lista dos táxons de insetos visitantes florais de Ocimum selloi, em Santa Cruz do Sul, RS: NI (número de indivíduos), D (dominância) e C (constância). Status: Ac (acessória); Ad (acidental); C (constante); Sd (subdominante); Rc (recessiva); Rr (rara); D (dominante); Ed (eudominante).

\begin{tabular}{ccccc}
\hline TÁXON & NI & D & C \\
\hline Hymenoptera & 370 & & \\
\hline
\end{tabular}

\section{Apoidea}

Apidae

Apis mellifera (Linnaeus, 1758)

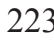

Bombus atratus (Franklin, 1913)

Bombus morio (Swederus, 1787)

Xylocopa sp. 1

Melissoptila sp. 1

Crabronidae sp. 1

Halictidae

Agapostemom sp. 1

2

Augochlora sp. 1

Augochlora sp. 2

Augochlorella ephyra (Schrottky, 1910)

Augochlorella urania (Smith, 1853)

Augochloropsis multiplex (Vachal, 1903)

Augochloropsis sp. 1

Ceratalictus sp. 1

Dialictus sp. 1

Dialictus sp. 2

Dialictus sp. 3

Halictillus sp. 1

Paraxistoglossa sp. 1

Megachilidae

Anthidium sp. 1

Anthidium sp. 2

Coelioxys sp. 1

$\begin{array}{lll}223 & \text { Ed } & \text { C } \\ 1 & \mathrm{Rr} & \mathrm{Ad} \\ 1 & \mathrm{Rr} & \mathrm{Ad} \\ 2 & \mathrm{Rr} & \mathrm{Ad} \\ 1 & \mathrm{Rr} & \mathrm{Ad} \\ 2 & \mathrm{Rr} & \mathrm{Ad}\end{array}$

C

Ad

Ad

Ad

Ad

Ad

Ad

C

Ad

Ad

Ac

Ac

C

Ac

C

Ac

Ad

Ad

Ad

$2 \mathrm{Rr}$

Ac

Ad

Ad 
Epanthidium sp. 1

1

Megachile sp. 1

Megachille sp. 2

2

1
1

Leucospidae sp. 1
$\mathrm{Rr}$

$\mathrm{Rr}$

$\mathrm{Rr}$

$\mathrm{Rr}$

$\mathrm{Rr}$
$\mathrm{Rr}$

$\mathrm{Rr}$
Ad

Ad

Ad

Ad

Ad

Ad

Ad
Evaniidae sp. 1

\section{Ichneumonoidea}

Braconidae sp. 1

Ichneumonidae

Campopleginae sp. 1

1

1

1

\section{Vespoidea}

Formicidae

Ponerinae sp. 1

Pseudomyrmicinae sp. 1

Pompilidae sp. 1

Vespidae

Pachodynerus guadulpensis (Saussure, 1853)

Polybia ignobilis (Haliday, 1836)

Polybia sericea (Oliver, 1792)

Synoeca cyanea (Fabricius, 1775)

Zeta argillaceum (Linnaeus, 1758)
1

1

1

4

1

3

1

3

\section{Tenthredinoidea}

Tentheredinidae sp. 1

\section{Coleoptera}

$\operatorname{Rr}$

Ad

31

Chrysomelidae

Diabrotica speciosa (Germar, 1824)

Curculionidae sp. 1

$\begin{array}{lll}13 & \mathrm{Sd} & \text { Ac } \\ 2 & \mathrm{Rr} & \text { Ad }\end{array}$

Lagriidae

Lagria villosa (Fabricius, 1781)

\section{Diptera}

$\mathrm{Sd}$

Ac

Asilidae

Blepharepium cajennensis (Fabricius, 1787)

Calliphoridae sp. 1

16

56

Culicidae sp. 1

$\begin{array}{ccc}4 & \mathrm{Rr} & \mathrm{Ac} \\ 2 & \mathrm{Rr} & \mathrm{Ad} \\ 2 & \mathrm{Rr} & \mathrm{Ad} \\ 1 & \mathrm{Rr} & \mathrm{Ad} \\ 28 & \mathrm{Sd} & \mathrm{C} \\ 2 & \mathrm{Rr} & \mathrm{Ad}\end{array}$

Culicidae sp. 2

Dolichopodidae sp. 1

2

$\mathrm{Rr}$

Ad

Ad

Ad

Ac

Ad

Ad

Ad

Ad

Micetophilidae sp. 1 
Micetophilidae sp. 2

Sarcophagidae sp. 1

$\mathrm{Rr}$

Ad

Syrphidae sp. 1

$\mathrm{Rr}$

Ac

Syrphidae sp. 2

$\mathrm{Rr}$

Ad

Syrphidae sp. 3

$\mathrm{Rr}$

Ad

Tachinidae sp. 1

$\mathrm{Rr}$

Ad

Tachinidae sp. 2

$\mathrm{Rr}$

Ad

Tachinidae sp. 3

$\mathrm{Rr}$

Ad

Tachinidae sp. 4

\section{Hemiptera}

$\mathrm{Rr}$

Ad

$\mathrm{Rr}$

Ad

Cicadellidae

Macugonalia sp. 1

Sibovia sagata (Signoret, 1854)

Cicadellidae sp. 1

163

Cicadellidae sp. 2

Cicadellidae sp. 3

$\begin{array}{ccc}48 & \mathrm{D} & \mathrm{C} \\ 4 & \mathrm{Rr} & \mathrm{Ad} \\ 6 & \mathrm{Rr} & \mathrm{Ac} \\ 1 & \mathrm{Rr} & \mathrm{Ad} \\ 1 & \mathrm{Rr} & \mathrm{Ad}\end{array}$

Cicadellidae

Ledrinae sp. 1

$\mathrm{Rr}$

Ac

Ledrinae sp. 2

3

Delphacidae sp. 1

2

Miridae sp. 1

1

Nabidae sp. 1

87

Nabidae sp. 2

3

Pentatomidae sp. 1

1

Pentatomidae sp. 2

Reduviidae sp. 1

Reduviidae sp. 2

$\mathrm{Rr}$

Ad

$\mathrm{Rr}$

Ad

Reduviidae sp. 3

Ed

C

$\mathrm{Rr}$

Ad

Scutelleridae sp. 1

\begin{tabular}{llccc}
\hline & Lepidoptera & $\mathbf{2 4}$ & & \\
Não determinado & & 24 & $\mathrm{Sd}$ & $\mathrm{C}$ \\
\hline & Odonata & $\mathbf{2 4}$ & & \\
\hline Coenagrionidae sp. 1 & & 1 & $\mathrm{Rr}$ & $\mathrm{Ad}$ \\
Libellulidae sp. 1 & & 2 & $\mathrm{Rr}$ & $\mathrm{Ad}$ \\
Protoneuridae sp. 1 & Orthoptera & 1 & $\mathrm{Rr}$ & $\mathrm{Ad}$ \\
\hline & & $\mathbf{5}$ & $\mathrm{Ar}$ & $\mathrm{Ad}$ \\
Acrididae sp. 1 & & 1 & $\mathrm{Rr}$ & $\mathrm{Ad}$ \\
Ommexechidae sp. 1 & & 2 & $\mathrm{Rr}$ & $\mathrm{Ad}$ \\
Tettigonidae sp. 1 & & 2 & $\mathrm{Rr}$ & \\
\hline
\end{tabular}


FIGURA 1: Relação entre as ordens e número de insetos coletados, em diferentes horários, nas inflorescências de Ocimum selloi, Santa Cruz do Sul, RS.

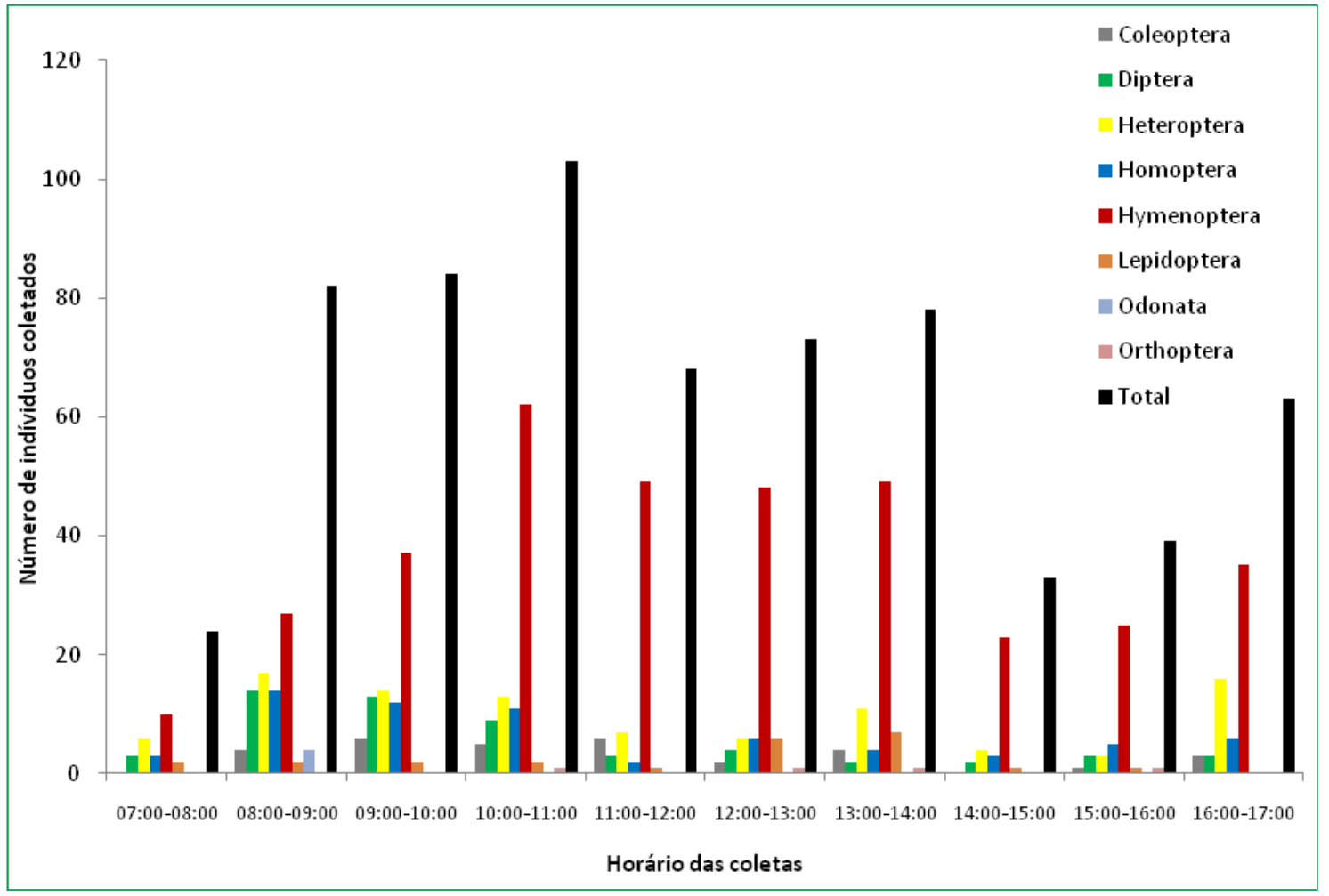

\section{Discussão}

No estudo de Silva et al. (2008), os principais visitantes florais de Ocimum canum (Sims), o popular manjericão-doce, são himenópteros dos gêneros Apis, Eulaema, Bombus, Augochloropsis e Megachile, sendo registrado ainda dípteros e lepidópteros. A atividade destes visitantes torna-se intensa durante todo o período da antese. Isto corrobora com os táxons coletados neste estudo para $O$. selloi.

Já em estudo realizado por Gonçalves et al. (2008), o qual analisou a atividade de insetos em flores de Ocimum gratissimum L., foram observadas 11 diferentes espécies visitando suas flores, prevalecendo as espécies da família Apidae, com predominância de $A$. mellifera, cuja maior frequência ocorreu no período das 7 ás $9 \mathrm{~h}$ da manhã. Ainda neste mesmo trabalho, verificou-se que a maior quantidade de insetos era da ordem Hymenoptera (famílias Apidae, Formicidade, Halictidae e Vespidae). Diferentemente dos dados obtidos por Gonçalves et al. (2008), o presente estudo demonstra que a maior freqüência tanto de $A$. mellifera quanto dos demais espécimes de Hymenoptera ocorreu no período das $10 \mathrm{~h}$. Além disso, A. mellifera foi a única espécie coletada às $7 \mathrm{~h}$ da manhã, devido à capacidade de manter a temperatura corporal alta, o que lhes garante o acesso às flores muito cedo, além de promover uma grande vantagem na competição por recursos florais em relação às abelhas nativas (PIRANI; CORTOPASSI-LAURINO, 1993).

Para Kevan e Baker (1983), a luminosidade influencia o horário das visitas de Apidae e Vespidae mais do que pela variação na temperatura. As características corporais e limitações fisiológicas dos visitantes florais podem gerar padrões de visitação por dependência de fatores como luminosidade e temperatura (RAMALHO et al., 1991). Faria (1994) observou que as baixas temperaturas, registradas nas primeiras horas da manhã e no final da tarde, correspondia a menor atividade das abelhas e vespas.

Além disso, a preferência de himenópteros por plantas do gênero Ocimum pode ser explicada pela 
presença de constituintes químicos como o eugenol e o cineol, os quais estão presentes, segundo Lorenzi e Matos (2002), desde as folhas até as flores da planta. Segundo Khosla (1986) as espécies da família Lamiaceae possuem uma estrutura floral favorável a polinização por abelhas, sendo A. mellifera considerado o polinizador mais comum do gênero Ocimum (DARRAH, 1980).

De acordo com Zanette et al. (2005) áreas mais urbanizadas são menos complexas e por isso ocorre uma menor abundância de vespas e abelhas, porém no presente estudo obteve-se uma alta diversidade de abelhas, tendo em vista que além da presença de prédios ao redor da área de estudo, existem diversas áreas abertas, propiciando aos visitantes florais do manjericão microhabitats de nidificação. Ao contráio, das espécies de Xylocopa e Bombus, que podem voar mais de $10 \mathrm{Km}$ para chegar até os recursos naturais, não necessitando de habitats de nidificação próximos (ROUBIK, 1992) e, portanto, encontradas em quantidades inferiores.

A alta densidade de prédios e falta de vegetação com árvores de grande porte reduz a ampla oferta de locais para nidificação de abelhas, principalmente para Meliponini como, por exemplo, de Plebeia ou Melipona, que fazem seus ninhos, geralmente, em árvores ocas, barrancos ou em outros locais, desde que os ocos sejam de tamanho apropriado e não aquecido pelo sol em demasia (ZANETTE et al. 2005; NOGUEIRA-NETO, 1970), justificando a ausência de espécies de Meliponini no manjericão investigado. Ao contrário, a grande diversidade de espécies de Halictidae deu-se pelo hábito da maioria das espécies em fazer seus ninhos no chão.

Através deste estudo, constatou-se que $A$. mellifera foi o principal visitante floral e um potencial polinizador de $O$. selloi, devido à sua elevada constância e dominância nas inflorescências, se comparada aos demais visitantes. De acordo com Oliveira e Cunha (2005) A. mellifera está bem adaptada em áreas urbanas e bordas de florestas e sua elevada densidade em determinadas espécies de flores pode influenciar a atividade de coletada por insetos nativos.

Os dados referentes à comunidade de visitantes florais de $O$. selloi obtidos neste estudo, fornecem informações básicas para pesquisas futuras sobre a biologia da polinização desta espécie como também para o gênero, tanto do ponto de vista científico como econômico.

\section{Referências}

ALMEIDA, O. S.; SILVA, A. H. B.; SILVA, A. B.; AMARAL C. L. F. Estudo da biologia floral e mecanismos reprodutivos do alfavacão (Ocimum officinalis L.) visando o melhoramento genético. Acta Scientiarum, Maringá, v. 26, n. 3, p. 343-348, 2004.

AZEVEDO FILHO, W. S. de; PRATES JUNIOR, P. H. de S. Técnicas de coleta e identificação de insetos. 2 ed. Porto Alegre: Cadernos EDIPUCRS - Série Zoologia no 4, 2005. 97 p.

BLANK, A. F.; CARVALHO FILHO, J. L. S.; SANTOS NETO, A. L.; ALVES, P. B.; ARRIGONI-BLANK, M. F.; SILVA-MANN, R.; MENDONÇA, M. C. Caracterização morfológica e agronômica de acessos do manjericão e alfavaca. Horticultura Brasileira, Brasília, v. 22, n. 1, p. 113-116, 2004.

BODENHEIMER, F. S. Precis d'écologie animal. Paris: Payot, $1955.315 \mathrm{p}$.

BORTOLI, C.; LAROCA, S. Melissocenologia no Terceiro Planalto Paranaense. I: Abundância relativa de abelhas silvestres (Apoidea) de um biótopo urbano de Guarapuava (PR, Brasil). Acta Biológica Paranaense, Curitiba. v. 26, p. 51-86, 1997.

COLLISCHONN, E. O Espaço natural na região do Vale do Rio Pardo - Algumas considerações. In: VOGT, O. P.; SILVEIRA, R. L. L. (Org.). Vale do Rio Pardo: (re)conhecendo a região. Santa Cruz do Sul: UNISC. 2001, p. 19-46.

COnStantinO, R.; DINIZ, I. R.; MOTTA, P. C. Textos de entomologia. Parte I: Biologia. Brasília: Universidade de Brasília, 2002. 93 p.

DARRAH, H. H. The cultivated basils. Independence: Buckeye Printing, 1980. 40 p.

FAEGRI, K.; VAN DER PIJL, L. The principles of pollination ecology. Oxford: Pergamon Press, 1980. 291 p.

FARIA, G. M. A flora e a fauna apícola de um ecossistema rupestre, Serra do Cipó - MG, Brasil: composição, fenologia e suas interações. 1994. 238 f. Tese (Doutorado em Biologia Vegetal)

- Universidade Estadual Paulista, Rio Claro. 1994.

FORTES, A. B. Compêndido de Geografia Geral do Rio Grande do Sul. 6. ed. Porto Alegre: Sulina, 1979, 246 p.

GINSBERG, H. S. Foraging ecology of bees in an old field. Ecology, New York, v. 1, p. 165-175, 1983.

GONÇALVES, C. B. S.; SILVA, C. B.; MOTA, J. H.; SOARES, T. $\mathrm{S}$. Atividade de insetos em flores de Ocimum gratissimum L. e suas interações com fatores ambientais. Revista Caatinga, Mossoró, v. 21, n. 3, p. 128-133, 2008

GRISWOLD, T.; PARKER, F. D.; HANSON, P. E. The bees (Apidae). In: HANSON, P. E.; GAULD, I. D. (Ed.). The Hymenoptera of Costa Rica. Oxford: Oxford University Press, 1995. p. 650-691.

IBGE - RADAMBRASIL. Levantamento de recursos naturais. Rio de Janeiro: IBGE, 1986. 791 p. 
JAVANMARDI, J.; KHALIGHI, A.; KASHI, A.; BAIS, H. P.; VIVANCO, J. M. Chemical characterization of basil (Ocimum basilicum L.) found in local accessions and used in traditional medicines in Iran. Journal of Agricultural and Food Chemistry, Washington, v. 50, p. 5878-5883, 2002.

KEVAN, P. G.; BAKER, H. G. Insects as flowers visitors and pollinators. Annual Review of Entomology, Palo Alto, v. 28, p. 407-453, 1983.

KHOSLA, M. K. Inter-relationship studies of different species the genus Ocimum. Journal of Plant Anatomy Morphology, Jodhpur, v. 3, p. 157-167, 1986.

KNOLL, F. R. N., BEGO, L. R.; IMPERATRIZ-FONSECA, V. L. I. As abelhas em áreas urbanas. Um estudo comparativo no Campus da Universidade de São Paulo. In: PIRANI, J. R.; CORTOPASSILAURINO, M. (Coord.) Flores e abelhas em São Paulo. São Paulo: FAPESP, EDUSP, 1993, p. 31-42.

KÖPPEN, W. Climatologia: con un estudio de los climas de la tierra. Fondo de Cultura Econômica. México, 1948, 479p.

LAROCA, S.; CURE, J. R.; BORTOLI, C. A associação de abelhas silvestres (Hymenoptera: Apoidea) de uma área restrita no interior da cidade de Curitiba (Brasil): Uma abordagem biocenótica. Duscenia, Curitiba, v. 13, p. 93-117, 1982.

LORENZI, H.; MATOS, F. J. A. Plantas medicinais no Brasil: nativas e exóticas cultivadas. Nova Odessa: Instituto Plantarum, 2002. 544 p.

MATOS, F. J. A. Farmácias vivas: sistema de utilização de plantas medicinais projetado para pequenas comunidades. 3. ed. Fortaleza: EUFC, 1998, 220 p.

MORALES, R. M.; SIMON, J. E. New basil selections with compact inflorescence of the ornamental market. In: JANICK, J. (Ed.). New crops. Alexandria: ASHS Press, 1996, p. 543-546.

MORHY, L. Metil-chavicol, cis e trans-anetol no óleo essencial de Ocimum selloi Benth. Anais da Academia Brasileira de Ciências, São Paulo, v. 45, n. 3/4, p. 401-412, 1973.

NOGUEIRA-NETO, P. A Criação de Abelhas Indígenas sem Ferrão Meliponinae. Chácaras e Quintais, São Paulo, 1970. 365 p.

OLIVEIRA, M. L.; CUNHA, J. A. Abelhas africanizadas Apis mellifera scutellata Lepeletier, 1835 (Hymenoptera: Apidae: Apinae) exploram recursos na floresta amazônica? Acta Amazônia, Manaus, v. 35, n. 3, p. 389-394, 2005.

PALISSA, A. E.; WIEDENROTH, M.; KLIMT, K. Anleitung zum ökologischen Geländepraktikum. Potsdam: Wissenschaftliches Zentrum der Pädagogischen Hochschule Potsdam, 1979, 186 p.
PATON, A. A synopsis of Ocimum L. (Labiatae) in Africa. Kew Bulletin, Kew, v. 47, n. 3, p. 403-435, 1992.

PIRANI, J. R.; CORTOPASSI-LAURINO, M. Flores e abelhas em São Paulo. São Paulo: EDUSP/FAPESP, 1993. 192 p.

PLEASANTS, J. M. Competition for bumblebee pollinators in Rocky Mountain Plant Communities. Ecology, New York, v. 6, p. 1446-1459, 1980.

RAMALHO, M.; IMPERATRIZ-FONSECA, V. L.; GIOVANNINI, A. K. Ecologia nutricional de abelhas sociais. In: PANIZZI, A. R.; PARRA, J. R. P. (Ed.). Ecologia nutricional de insetos e suas implicações no manejo de pragas. Brasília/Barueri: $\mathrm{CNPq} / \mathrm{Ed}$. Manole Ltda., 1991. p. 225-252.

RAJU, A. J. S. Pollination ecology of Ocimum americanum L. and $O$. basilicum L. (Lamiaceae) in India. Plant Species Biology, Sapporo, v. 4, p. 107-116, 1989.

ROUBIK, T. W. Ecology and Natural History of Tropical Bees. Cambridge University Press, Cambridge, 1992, 514p.

SILVA, A. B.; SOUZA, M. F.; DA SILVA, A. H. B.; ALMEIDA, O. da S.; DA SILVA, A. B.; AMARAL, C. L. F. Biologia floral e mecanismos reprodutivos de Ocimum canum Sims (Lamiaceae). Biotemas, Florianópolis, v. 21, n. 2, p. 33-40, 2008.

SILVEIRA, F. A.; MELO G. A. R.; ALMEIDA A. B. Abelhas brasileiras: sistemática e identificação. Belo Horizonte: IDM, 2002. 253 p.

SIMON, J. E.; QUINN, J.; MURRAY, R. G. Basil: a source of essential oils. In: JANICK, J.; SIMON, J. E. (Ed.). Advances in new crops. Portland: Timber Press, 1990. p. 484-489.

TRIPLEHORN, C. A.; JOHNSON, N. F. Estudo dos insetos. São Paulo: Cengage Learning, 2011, 809p.

VINCENT, C. R.; JACOBI, C. M.; ANTONINI, Y. Diversidade na adversidade. Ciência Hoje, Rio de Janeiro, v. 31, p. 64-67, 2002.

ZANETTE, L. R. S.; MARTINS, R. P.; RIBEIRO, S. P. Effects os urbanization on Neotropical wasp and bee assemblages in a Brazilian metropolis. Landscape and Urban Planning, San Diego, v. 71, p. 105-121, 2005.

WILMS, W.; WENDEL, L.; ZILLIKENS, A.; BLOCHTEIN, B.; ENGELS, W. Bees and other insects recorded on flowering trees in a subtropical Araucaria forest in southern Brazil. Studies on Neotropical Fauna and Environment, Tübingen, v. 32, p. 220 226, 1997. 